\title{
Aproximación genética en la esclerosis lateral amiotrófica
}

\author{
Iván Cervantes-Aragón, ${ }^{1}$ Sergio Alberto Ramírez-García, ${ }^{2,3}$ Luz Margarita Baltazar-Rodríguez, ${ }^{3}$ \\ Diana García-Cruz $z^{4 *}$ y Gema Castañeda-Cisneros ${ }^{5}$ \\ 'Universidad Guadalajara Lamar, Guadalajara, Jalisco; ${ }^{2}$ Sistema de Universidades de Oaxaca, Instituto de Investigaciones Sobre la Salud Pública, \\ Universidad de la Sierra Sur, Oaxaca; ${ }^{3}$ Universidad de Colima, Facultad de Medicina, Colima; ${ }^{4}$ Universidad de Guadalajara, Centro Universitario \\ de Ciencias de la Salud, Departamento de Biología Molecular y Genómica, Jalisco; ${ }^{5}$ nstituto Mexicano del Seguro Social, Centro Médico Nacional \\ de Occidente, Servicio de Neurocirugía, Jalisco. México
}

\section{Resumen}

El gen SOD1 es el primer gen responsable mapeado en la esclerosis lateral amiotrófica tipo 1 (ELA1) y codifica para la enzima superóxido dismutasa tipo 1 (SOD1), cuya función es proteger del daño mediado de los radicales libres derivados del oxígeno; su mecanismo fisiopatológico en ELA1 se relaciona con isquemia. Diversos estudios moleculares del gen SOD1 muestran que las mutaciones puntuales son las más frecuentes. Las mutaciones más comunes en los casos familiares son p.A4V, p.I113Y, p.G37R, p.D90A y p.E100G, que explican más de $80 \%$ de los casos, aunque también se han descrito mutaciones intrónicas como responsables de esclerosis lateral amiotrófica tipo 1. Los casos esporádicos se explican por mutaciones en otros genes como SETX y C9orf72. ELA1 es una enfermedad compleja con heterogeneidad genética. Por otra parte, los casos familiares y esporádicos tienen etiología distinta, lo cual se explica por la heterogeneidad molecular y múltiples mecanismos patogénicos que conducen a ELA1; el estrés oxidativo y la isquemia no son la única causa. En México son escasos los estudios de genética molecular de esclerosis lateral amiotrófica. Los estudios clínicos muestran incremento de citocinas como la adipsina en el líquido cefalorraquídeo.

PALABRAS CLAVE: Enfermedad de la neurona motora familiar. Atrofia muscular. Superóxido dismutasa 1.

\section{Abstract}

The superoxide dismutase type 1 (SOD1) gene is the first responsible gene mapped in amyotrophic lateral sclerosis type 1 (ALS1), and it codes for the enzyme SOD1, the function of which is to protect against damage mediated by free radicals deriving from oxygen. Its pathophysiological mechanism in ALS1 is related to ischemia. Several molecular studies of the SOD1 gene show that point mutations are the most frequent. The most common mutations in familial cases are p.A4V, p.I113Y, p.G37R, p.D90A and p.E100G, which account for more than $80 \%$ of cases, although intronic mutations have also been described as responsible for ALS1. Sporadic cases are explained by mutations in other genes such as SETX and C9orf72. ALS1 is a complex disease with genetic heterogeneity. On the other hand, familial and sporadic cases have a different etiology, which is explained by molecular heterogeneity and multiple pathogenic mechanisms that lead to ALS1; oxidative stress and ischemia are not the only cause. In Mexico, ALS molecular genetics studies are scarce. Clinical studies show an increase in cytokines such as adipsin in cerebrospinal fluid.

KEY WORDS: Familial motor neuron disease. Muscle atrophy. Superoxide dismutase 1.

Correspondencia:

*Diana García-Cruz

E-mail: dianagarcr@hotmail.com
Fecha de recepción: 13-12-2018

Fecha de aceptación: 21-02-2019

DOI: 10.24875/GMM.19004927
Gac Med Mex. 2019;155:513-521

Disponible en PubMed www.gacetamedicademexico.com 


\section{Introducción}

La esclerosis lateral amiotrófica (ELA) es una enfermedad neurodegenerativa progresiva letal que afecta a las neuronas motoras superior e inferior. ${ }^{1-2}$ La prevalencia en México es de 5000 a 7000 pacientes según Martínez et al. En Francia se estima 2.5 por 100000 habitantes. ${ }^{3}$ La edad de inicio es de aproximadamente 47.5 años, con supervivencia hasta de 58.9 meses posteriores al diagnóstico. Otero et al. reportaron que la ELA representa más de $90 \%$ de los casos de las enfermedades de la motoneurona en México, el fenotipo incluye signos de neurona motora superior o inferior. ${ }^{1}$

\section{Epidemiología genética y molecular}

La ELA se presenta esporádicamente (ELAE) en 90 a $95 \%$ de los casos y de forma familiar (ELAF) en 5 a $10 \%$. Las formas esporádicas se observan más fuera del continente europeo.1-3 Las formas de ELAF se asocian con gran número de genes pleiotrópicos (Tabla 1), lo que resulta en superposición de fenotipos clínicos y patológicos. También se han descrito diferentes patrones de herencia. ${ }^{3}$ Los genes más frecuentemente asociados con ELAF son SOD1 Tau. ${ }^{4,5}$ Las mutaciones en el gen SOD1 explica $2 \%$ de afectados por ELAE, de las cuales aproximadamente $10 \%$ se heredan como un patrón autosómico dominante con

Tabla 1. Tipos de ELA de acuerdo con el gen afectado y su patrón de herencia

\begin{tabular}{|c|c|c|c|c|c|c|}
\hline Tipo & Símbolo & Gen & Locus & ELAF & ELAE & Herencia \\
\hline ELA 1 & SOD1 & Cu/Zn superóxido dismutasa 1 & $21 q 22.11$ & Sí & Sí & $A D$ y $A R$ \\
\hline ELA 2 & ALS2 & Alsina 2 & $2 q 33.2$ & Sí & No & AR \\
\hline ELA 3 & ALS3 & Alsina 3 & $18 q 21$ & Sí & No & $A D$ \\
\hline ELA 4 & SETX & Senataxina & $9 q 34.13$ & Sí & Sí & $A D$ \\
\hline ELA 5 & SPAST & Espastina & $2 p 24$ & Sí & Sí & $\mathrm{AR}$ \\
\hline ELA 6 & FUS & Gen derivado de $t(12 ; 16)$ & $16 \mathrm{p} 11.2$ & Sí & Sí & $A D$ \\
\hline ELA 7 & ALS7 & Alsina 7 & 20p13 & Sí & No & $A D$ \\
\hline ELA 8 & $V A P B$ & $\begin{array}{l}\text { Proteína B asociada con la proteína asociada con } \\
\text { membrana de vesícula }\end{array}$ & $20 q 13.33$ & Sí & No & $A D$ \\
\hline ELA 9 & ANG & Angiogenina & $14 q 11.1$ & Sí & Sí & $A D$ \\
\hline ELA 10 & TARDBP & Proteína de unión TAR DNA & $1 \mathrm{p} 36.22$ & Sí & Sí & $A D$ \\
\hline ELA 11 & FIG4 & $\begin{array}{l}\text { Homólogo a FIG4, dominio contenedor de fosfatasa } \\
\text { lipídica SCA1 }\end{array}$ & $6 q 21$ & Sí & Sí & $A D$ \\
\hline ELA 12 & OPTN & Optineurina & $10 p 13$ & Sí & Sí & $A D Y A R$ \\
\hline ELA 13 & ATXN2 & Ataxina 2 & $12 q 23-24.1$ & No & Sí & --- \\
\hline ELA 14 & VCP & Proteína contenedora de valiosina & $9 p 13$ & Sí & No & $A D$ \\
\hline ELA 15 & UBQLN2 & Ubiquitina 2 & Xp11.21 & Sí & No & Ligado a X dominante \\
\hline ELA 16 & SIGMAR1 & Receptor intracelular no opioide sigma 1 & $9 p 13$ & Sí & No & AR \\
\hline ELA 17 & CHMP28 & ----- & $3 p 11.2$ & No & Sí & --- \\
\hline ELA 18 & PFN1 & Profilina 1 & $17 p 13.3$ & No & Sí & --- \\
\hline ELA 19 & ERBB4 & $\begin{array}{l}\text { Homólogo del oncogen de la leucemia viral } \\
\text { eritroblástica aviar }\end{array}$ & $2 q 34$ & Sí & No & $A D$ \\
\hline ELA 20 & HNRNPA1 & Ribonucleoproteína A1 heterogénea nuclear & $12 q 13.13$ & Sí & Sí & $A D$ y $A R$ \\
\hline ELA 21 & MATR3 & Matrina 3 & $5 q 31.2$ & Sí & No & $A D$ \\
\hline ELA-DFT2 & $\mathrm{CHCHD10}$ & $\begin{array}{l}\text { Coiled-Coil-Helix-Coiled-Coil-Helix } \\
\text { Domain-Containing Protein } 10\end{array}$ & $9 q 21-q 22$ & Sí & No & $A D$ \\
\hline
\end{tabular}


Tabla 1. Tipos de ELA de acuerdo con el gen afectado y su patrón de herencia (Continuación)

\begin{tabular}{|c|c|c|c|c|c|c|}
\hline Tipo & Símbolo & Gen & Locus & ELAF & ELAE & Herencia \\
\hline ELA-DFT1 & C9orf7 2 & Marco de lectura abierto 72 del cromosoma 9 & $9 p 21.2$ & Sí & Sí & $A D$ \\
\hline Riesgo & UNC13A & Homólogo de Caenorhabditis eleganss A & $19 p 13.11$ & No & Sí & \\
\hline---- & $D A O$ & D-amino ácido oxidasa & $12 q 24$ & Sí & No & $A D$ \\
\hline---- & DCTN1 & Dinactina & $2 \mathrm{p} 13$ & Sí & Sí & $A D$ \\
\hline---- & NEFH & $\begin{array}{l}\text { Cadena pesada de } 200 \text { kDA constitutiva de } \\
\text { neurofilamento }\end{array}$ & $22 q 12.1$ & Sí & Sí & $A D$ \\
\hline--- & PRPH & Periferina & $12 q 12$ & No & Sí & --- \\
\hline---- & SQSTM1 & Secuestrosoma 1 & $5 q 35$ & Sí & Sí & $A D$ \\
\hline---- & TAF15 & Factor asociado con proteína de unión a caja TATA & $17 q 11.1-q 11.2$ & Sí & No & $A D$ \\
\hline---- & SPAST & Espastina & $2 p 24$ & No & Sí & ---- \\
\hline---- & ELP3 & $\begin{array}{l}\text { Proteína de elongación homóloga } \\
3 \text { (Saccharomyces cerevisiae) }\end{array}$ & $8 p 21.1$ & No & Sí & --- \\
\hline---- & LMNB1 & Laminina B1 & $5 q 23.2$ & Sí & No & $A D$ \\
\hline
\end{tabular}

ELA = esclerosis lateral amiotrófica, ELAE = esclerosis lateral amiotrófica esporádica, ELAF = esclerosis lateral amiotrófica familiar, $A D=$ autosómica dominante, $A R=$ autosómica recesiva. ---- Se utiliza en la columna tipo cuando a pesar de que el gen estuvo asociado, no ha tenido un número de reportes suficiente para asignarle un nombre específico a la enfermedad asociada con él; en la columna herencia se utiliza para hacer notar que no se ha determinado un modo de herencia específico. Cuadro elaborado con base en los datos de OMIM y http://alsod.iop.kcl.ac.uk/

alta penetrancia después de la sexta década. El fenotipo clínico es similar en los casos de ELAF y ELAE. ${ }^{6-9}$ Cabe señalar que la mutación p.D90A de los escandinavos se trasmite con un patrón recesivo ${ }^{10}$ (Tabla 1).

Las mutaciones clásicas de SOD1 en ELA son p.G37T, p.L38V, p.G41S, p.G41D, p.H43R, p.G85R, p.G93C, p.G93A, p.E100G, p.L106V y p.I113Y, las cuales producen cambios en $S O D 1$, disminuyendo la actividad enzimática. ${ }^{7}$ En población italiana, en siete de 39 pacientes con ELAF de fenotipo atípico se encontraron las mutaciones p.G12R, p.G41S, p.L114F y p.D90A. Además, se identificó una variante p.S59S sinónima en un paciente con ELAE. ${ }^{7}$

En otra serie de casos, en 17 de 264 pacientes (6.4\%) se encontró el polimorfismo nt34A>C intrón 3 $y$ en un paciente ELAF fue identificada la variante IVS3+62 T>C. La frecuencia total de mutaciones del gen SOD1 (17.9\%) en casos de ELAF fue comparable con la encontrada en otros estudios, con un tamaño de muestra similar de casos de ELA. Entre los casos de ELAF, la mutación más frecuente fue p.G41S. ${ }^{11}$

En otra cohorte transversal de población italiana, ocho de 38 pacientes $(21 \%)$ con ELAF y cinco de 175 (3\%) con ELAE tenían mutaciones sin sentido en el gen SOD1. Se identificaron dos mutaciones más, una en el exón 4 (p.L84F) en un caso familiar y la segunda en el exón 3 (p.G72S) en un paciente esporádico. ${ }^{12}$

\section{El papel de la enzima SOD1}

El gen SOD1 tiene su locus en 21q22.11, presenta una longitud de 9310 pares de bases (pb) y se compone de cinco exones, codifica para la enzima superóxido dismutasa (SOD1). Esta proteína tiene 153 residuos de aminoácidos con un peso de $16 \mathrm{kDa}^{13}$ y actividades oxidorreductasa y peroxidasa. Su estructura cuaternaria está formada por hojas beta en forma de barril y en llave griega. ${ }^{14} \mathrm{Cada}$ homodímero constituye una unidad denominada A, B, C, D o E y tiene la capacidad de asociarse con otras cuatro unidades, alcanzando una conformación similar a un "hueso fémur de perro"; estas estructuras a su vez tienden a agruparse formando unidades asimétricas, las cuales generan una estructura similar a un "panal de abejas".14

La enzima SOD1 es citoplasmática, cataliza la conversión del superóxido con óxido nítrico (NO) a la forma del anión peroxidado (ONOO-), que genera radicales tóxicos $\mathrm{HO}$ y $\mathrm{NO}_{2}{ }^{14-16}$ Este mecanismo podría inducir la neurodegeneración debido a la acumulación de radicales libres causando muerte celular. ${ }^{7}$

Mediante inmunohistoquímica se ha demostrado que SOD1 se encuentra distribuida abundantemente en neuronas motoras, interneuronas y neuronas sensoriales en la médula espinal, ${ }^{17}$ con un patrón puntiforme en las porciones motoras y sensitivas de los núcleos de los nervios craneales, el soma, así como en dendritas proximales y axones terminales en individuos no 
Gaceta Médica de México. 2019;155

Tabla 2. Correlación genotipo-fenotipo para las mutaciones en SOD1

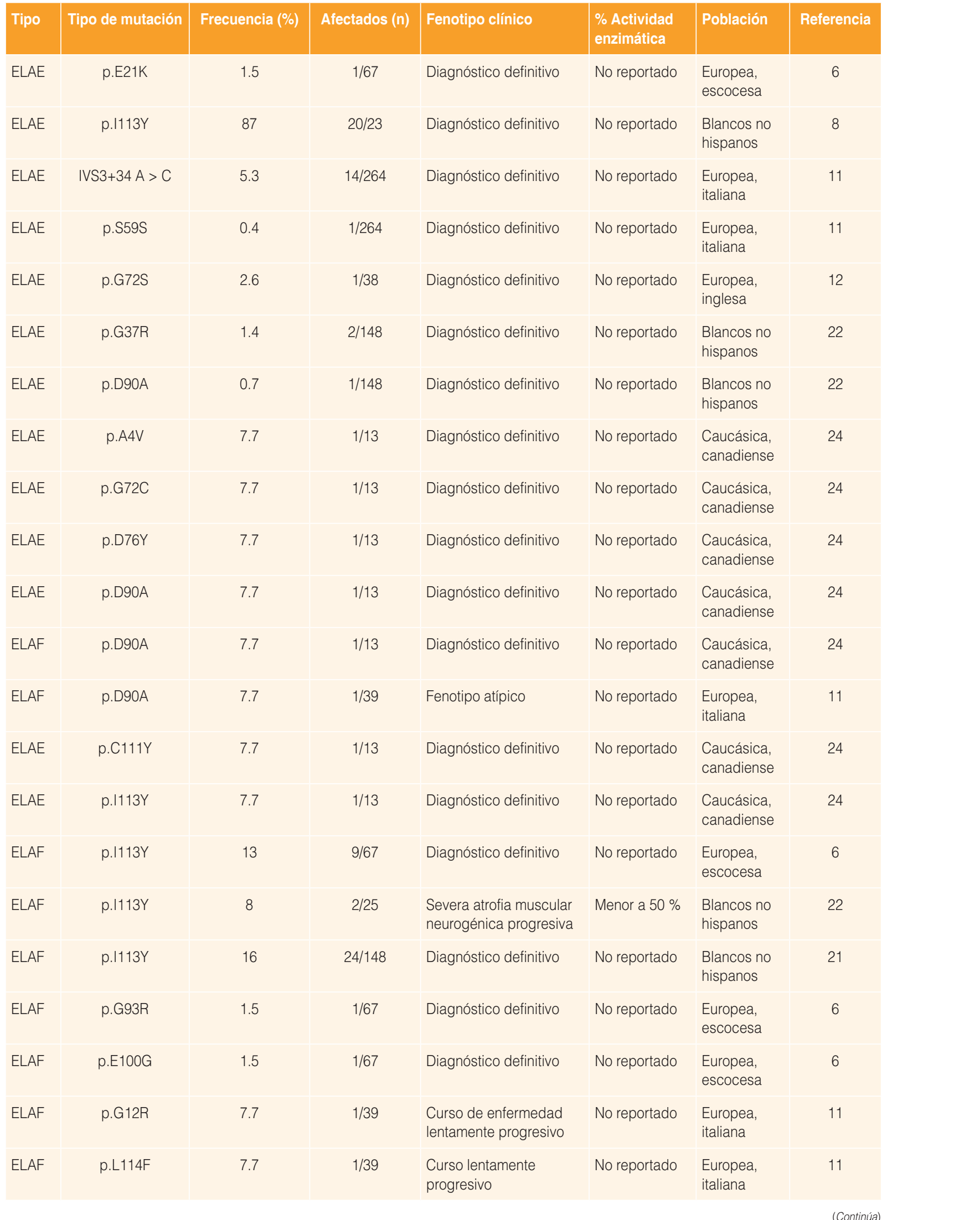


Tabla 2. Correlación genotipo-fenotipo para las mutaciones en SOD1 (Continuación)

\begin{tabular}{|c|c|c|c|c|c|c|c|}
\hline Tipo & Tipo de mutación & Frecuencia (\%) & Afectados (n) & Fenotipo clínico & $\begin{array}{l}\text { \% actividad } \\
\text { enzimática }\end{array}$ & Población & Referencia \\
\hline ELAF & IVS3+62 T > C & 0.4 & $1 / 14$ & Diagnóstico definitivo & No reportado & $\begin{array}{l}\text { Europea, } \\
\text { italiana }\end{array}$ & 11 \\
\hline ELAF & p.G72C & 7.7 & $1 / 13$ & Diagnóstico definitivo & No reportado & $\begin{array}{l}\text { Caucásica, } \\
\text { canadiense }\end{array}$ & 24 \\
\hline ELAF & p.D76Y & 7.7 & $1 / 13$ & Diagnóstico definitivo & No reportado & $\begin{array}{l}\text { Caucásica, } \\
\text { canadiense }\end{array}$ & 24 \\
\hline ELAF & p.C111Y & 7.7 & $1 / 13$ & Diagnóstico definitivo & No reportado & $\begin{array}{l}\text { Caucásica, } \\
\text { canadiense }\end{array}$ & 24 \\
\hline ELAF & p.G37R & 8 & $2 / 25$ & $\begin{array}{l}\text { Severa atrofia muscular } \\
\text { neurogénica progresiva }\end{array}$ & Menor a $50 \%$ & $\begin{array}{l}\text { Blancos no } \\
\text { hispanos }\end{array}$ & 22 \\
\hline ELAF & p.G93A & 8 & $2 / 25$ & $\begin{array}{l}\text { Severa atrofia muscular } \\
\text { neurogénica progresiva }\end{array}$ & Menor a $50 \%$ & $\begin{array}{l}\text { Blancos no } \\
\text { hispanos }\end{array}$ & 22 \\
\hline ELAF & p.E100G & 8 & $2 / 25$ & $\begin{array}{l}\text { Severa atrofia muscular } \\
\text { neurogénica progresiva }\end{array}$ & Menor a $50 \%$ & $\begin{array}{l}\text { Blancos no } \\
\text { hispanos }\end{array}$ & 22 \\
\hline ELAF & p.L84V & 10 & $3 / 30$ & Edad de inicio temprana & Menor a $50 \%$ & $\begin{array}{l}\text { Asiática } \\
\text { japonesa }\end{array}$ & 23 \\
\hline ELAF & p.H46R & 6.7 & $2 / 30$ & Curso clínico benigno & Menor a $50 \%$ & $\begin{array}{l}\text { Asiática } \\
\text { japonesa }\end{array}$ & 23 \\
\hline ELAF & p. $1104 \mathrm{~F}$ & 6.7 & $2 / 30$ & $\begin{array}{l}\text { Oftalmoparesia y } \\
\text { afectación sensorial }\end{array}$ & $\begin{array}{l}\text { Severa } \\
\text { reducción, } \\
\text { menor a } 10 \%\end{array}$ & $\begin{array}{l}\text { Asiática } \\
\text { japonesa }\end{array}$ & 23 \\
\hline ELAF & p.A4V & 15.28 & $2 / 13$ & Diagnóstico definitivo & No reportado & $\begin{array}{l}\text { Caucásica, } \\
\text { canadiense }\end{array}$ & 24 \\
\hline ELAF & p.I113Y & 13 & $3 / 23$ & Diagnóstico definitivo & No reportado & $\begin{array}{l}\text { Blancos no } \\
\text { hispanos }\end{array}$ & 8 \\
\hline ELAF & IVS3+34 A > C & 1.14 & $3 / 264$ & Diagnóstico definitivo & No reportado & $\begin{array}{l}\text { Europea, } \\
\text { italiana }\end{array}$ & 11 \\
\hline ELAF & p.G41S & 30.8 & $4 / 39$ & $\begin{array}{l}\text { Curso rápidamente } \\
\text { progresivo con deterioro } \\
\text { cognitivo severo }\end{array}$ & No reportado & $\begin{array}{l}\text { Europea, } \\
\text { italiana }\end{array}$ & 11 \\
\hline ELAF & p.V148I & 13.3 & $4 / 30$ & Inicio temprano & Menor a $10 \%$ & $\begin{array}{l}\text { Asiática } \\
\text { japonesa }\end{array}$ & 23 \\
\hline ELAF & p.A4V & 32 & $8 / 25$ & $\begin{array}{l}\text { Severa atrofia muscular } \\
\text { neurogénica progresiva }\end{array}$ & Menor a $50 \%$ & $\begin{array}{l}\text { Blancos no } \\
\text { hispanos }\end{array}$ & 22 \\
\hline ELAF & p.G41S & 100 & $9 / 9$ & $\begin{array}{l}\text { Fenotipo severo, } \\
\text { rápidamente progresivo }\end{array}$ & No reportado & $\begin{array}{l}\text { Europea, } \\
\text { italiana }\end{array}$ & 26 \\
\hline ELAF & p.D90A & 38.5 & $12 / 60$ & Diagnóstico definitivo & No reportado & $\begin{array}{l}\text { Asiática, } \\
\text { iraní }\end{array}$ & 25 \\
\hline ELAF & p.L84F & 2.6 & $1 / 38$ & Diagnóstico definitivo & No reportado & $\begin{array}{l}\text { Europea, } \\
\text { inglesa }\end{array}$ & 12 \\
\hline ELAF & p.A4V & 41 & $61 / 148$ & Diagnóstico definitivo & Menor a $10 \%$ & $\begin{array}{l}\text { Blancos no } \\
\text { hispanos }\end{array}$ & 21 \\
\hline
\end{tabular}

ELAE = esclerosis lateral amiotrófica esporádica, ELAF = esclerosis lateral amiotrófica familiar.

afectados: ${ }^{18}$ se ha encontrado difusamente en la corteza, hipocampo y amígdala. ${ }^{19}$ Por otro lado, se ha demostrado una expresión más abundante de SOD1 en pacientes afectados por ELA. Estas evidencias apoyan contundentemente la hipótesis de la ganancia de función con efecto tóxico y no haploinsuficiencia. ${ }^{18,20}$ 


\section{Mutaciones en el gen SOD1}

La mayoría de las mutaciones reportadas en SOD1 son cambios de nucleótido con sentido o por corrimiento del marco de lectura en intrones. ${ }^{1-3} \mathrm{El}$ efecto del porcentaje de la actividad enzimática es un campo poco estudiado, limitando la correlación genotipo-fenotipo. Entre los trabajos más extensos que reportan la frecuencia de mutaciones en el gen SOD1 está la cohorte realizada en blancos no hispanos en 2045 pacientes con ELAF y ELAE, de los cuales se encontraron mutaciones de SOD1 en 148 casos. Las mutaciones más prevalentes asociadas con la enfermedad en el gen SOD1 fueron p.A4V, en $41 \%$ de los pacientes, p.lle113Tre en $16 \%$ y mutaciones intrónicas en $11 \%$. Se encontraron 16 mutaciones exónicas (p.K8V, p.F20C, p.Q22L, p.H48R, p.T54R, p.S59I, p.V87A,

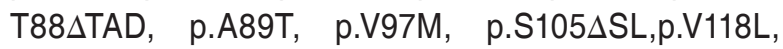
p.D124G, p.G141X, p.G147R y p.I151S, que corresponden a una frecuencia de $10.81 \%$. En $2.7 \%$ los casos con ELAE se detectaron cuatro pacientes con las siguientes mutaciones: p.G37R, p.D90A y p.E100G. ${ }^{21}$

En otra cohorte en blancos no hispanos con ELAF, la mutación p.A4V en el exón 1 fue la más frecuente (32 \%). Por otra parte, las mutaciones p.G37R, p.G93A, p.E100G y p.I113Y tuvieron una frecuencia de $8 \%$. El estudio de acoplamiento molecular (docking) de estas variantes revela la alteración de las interacciones para el contacto de los dobles y el dímero del barril beta. Los glóbulos rojos de los casos índices heterocigotos tenían menos de $50 \%$ de actividad, consistentes con un dímero de SOD estructuralmente defectuoso. ${ }^{22}$

En la clínica son importantes los estudios de correlación genotipo-fenotipo (Tabla 2), por ejemplo, en una cohorte de ELAF en población asiática japonesa se reportaron cuatro diferentes mutaciones con sentido en los exones 2, 4 y 5 del gen SOD1 en cinco familias: p.H46R, p.L84V, p.I104F y p.V148I. Los pacientes con la mutación p.H46R mostraron un curso clínico benigno y progresión estereotipada de debilidad muscular $\mathrm{y}$ atrofia de las piernas. Los portadores de p.L84V tienen un curso clínico de la enfermedad muy similar, la edad de inicio fue más joven en hombres que en mujeres. Los pacientes con p.I104F mostraron amplios rangos de edad de inicio y duración, con oftalmoparesia y afectación sensorial en un paciente. Aquellos con la mutación p.V148I mostraron una edad más temprana al inicio y primeros síntomas variables dentro de la familia. Aunque el signo NMI fue evidente en todos los casos, la hiperreflexia varió entre los pacientes con las diferentes mutaciones..$^{23}$ La parálisis bulbar fue frecuente en los portadores p.I104F, pero no en aquellos con p.H46R. La actividad de SOD1 de los glóbulos rojos se redujo severamente con p.I104F y p.V148I, pero se redujo ligeramente con p.H46R. ${ }^{24}$

Otro estudio con este enfoque en 254 pacientes con ELAF y ELAE de la provincia de Columbia Británica, Canadá, mostró en 13 pacientes (5.1\%) las mutaciones p.A4V, p.G72C, p.D76Y, p.D90A, p.C111Y y p.I113T en SOD1, tanto en ELAF y ELAE. Hubo discordancias clínicas incluso entre pacientes con la misma mutación. Lo anterior apoya a la hipótesis de que ELA es un trastorno heterogéneo en el que la genética, el ambiente y el envejecimiento se interrelacionan para formar el fenotipo clínico final. ${ }^{24}$

En una cohorte asiática iraní de 60 pacientes con ELA (cuatro familias) se encontraron mutaciones SOD1 en $11.7 \%$, $38.5 \%$ de los probandos ELAF y $4.25 \%$ de los casos ELAE; el cribado identificó homocigotos p.D90A en todas las familias vinculadas. El análisis de haplotipos sugiere que los pacientes iraníes podrían compartir un fundador común con el famoso alelo p.D90A escandinavo recesivo. Otras mutaciones identificadas fueron p.L84F, p.A4Y y p. $1113 Y .{ }^{25}$ En población italiana, un caso de ELAF se encontró con la mutación heterocigótica p.L106, cuya clínica se caracterizó por edad de inicio relativamente temprano, afección bulbar y espinal. ${ }^{25} \mathrm{El}$ mecanismo patogénico SOD1 en la muerte neuronal varía; líneas de evidencia sugieren un mal plegamiento de proteínas. El docking de la mutación p.G10R mostró fuerte desestabilización que influye en la fuerza de la interfaz del dímero, generando agregados intracelulares tóxicos, lo que apoya esta teoría. ${ }^{25}$

En un reporte de caso de dos pacientes italianos con ELA, aparentemente esporádicos, heterocigotos para la mutación p.D90A en SOD1, un paciente experimentó una afectación sensorial temprana. ${ }^{26}$ En seis familias con ELA, mediante secuenciación directa se detectó la mutación p.G41S. El patrón clínico de estos pacientes se caracterizó por la aparición espinal de motoneuronas superior e inferior con afectación temprana, aparición de signos bulbares en un año y muerte unos meses después; la edad promedio al inicio fue de 49.3 años y la duración promedio de la enfermedad fue de 0.9 años; se encontró un haplotipo común para los portadores de la mutación, lo cual evidencia un efecto fundador en Italia. Ciertamente la mutación p.G41S se relaciona constantemente con un fenotipo severo, rápidamente progresivo. ${ }^{26}$

De los estudios mencionados se obtuvieron los siguientes datos: el número total de individuos afectados 


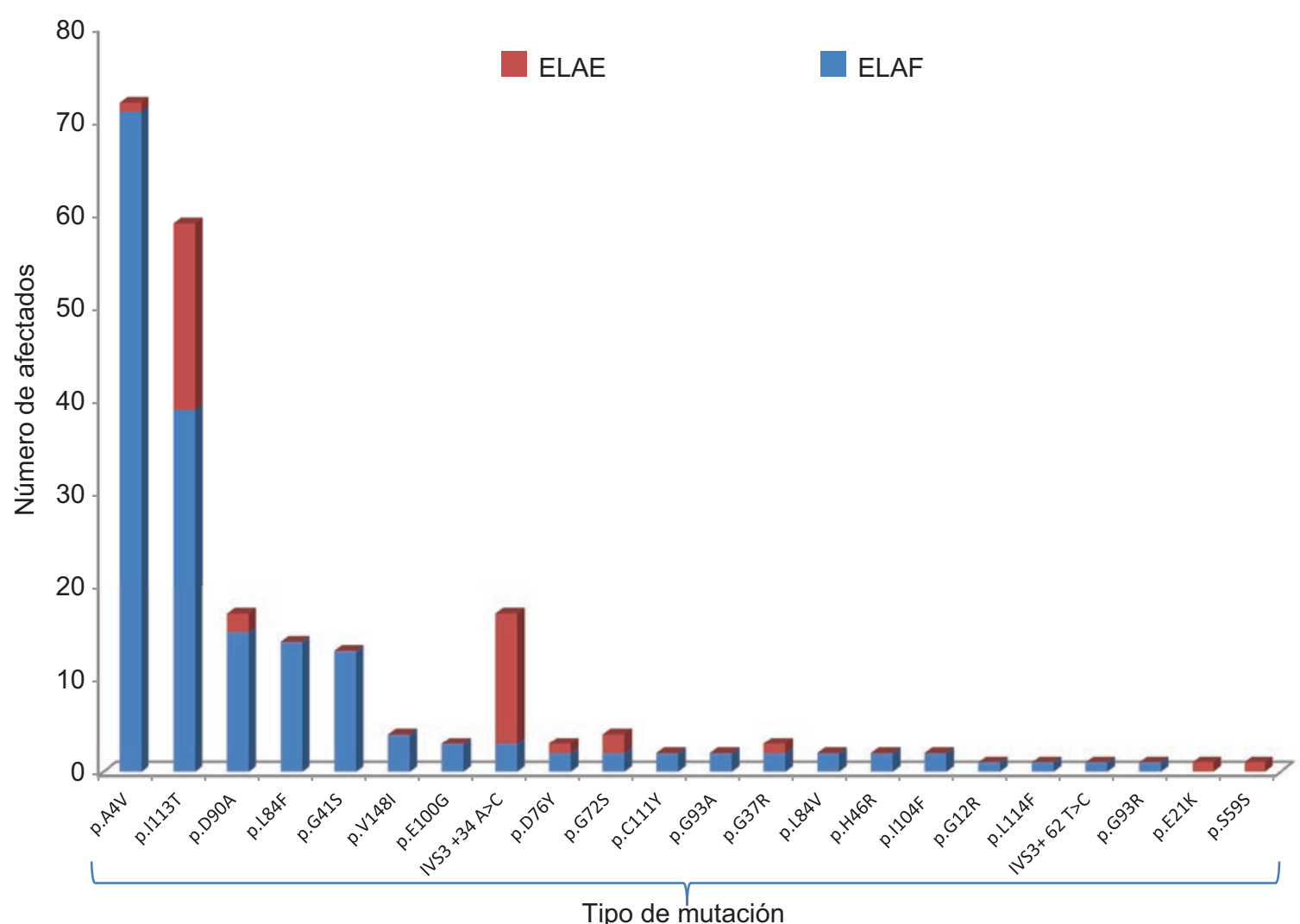

Figura 1. Proporción de mutaciones encontradas en el gen SOD1 en pacientes con esclerosis lateral amiotrófica tipo 1. ELAE = esclerosis lateral amiotrófica esporádica, ELAF = esclerosis lateral amiotrófica familiar.

por ELA fue de 2553, de los cuales 46 eran ELAE, en estos casos las mutaciones más frecuentes fueron p.I113T en $45.67 \%$ e IVS3+34 A>C en $30.44 \%$ (Figura 1). En los casos de ELAF, las mutaciones p.A4V, p.I113Y, p.D90A, p.L84F y p.G41S, con 39.01, 21.43, $8.24,7.69$ y $7.14 \%$, respectivamente. Únicamente dos de los estudios revisados analizan la función enzimática con las mutaciones encontradas en la población afectada. $^{22-23}$

\section{ELA una enfermedad compleja}

Los estudios genéticos muestran que la ELA es una enfermedad compleja por la heterogeneidad molecular; existen más de 23 genes responsables que tienen efecto directo sobre el fenotipo, como se describe en la Tabla 1. Se han reportado más de 140 mutaciones diferentes distribuidas en los cinco exones e intrones del gen SOD1, siendo p.A4V la mutación más frecuente en los casos familiares, mientras que en los casos esporádicos es p.1113T. ${ }^{10}$ Esto apoya al alto componente poligénico, heterogeneidad molecular y alélica que tiene ELA.
La presentación de la enfermedad varía, hay formas esporádicas y variantes familiares que se trasmiten con un patrón de herencia autosómico dominante, ${ }^{6-10}$ sin embargo, hay mutaciones que se trasmiten con un patrón recesivo. ${ }^{12,26,27}$ Incluso se han reportado individuos de 85 años portadores de la mutación p.D90A totalmente asintomáticos, esto aunado a la hipótesis sobre la existencia de un mecanismo complementario responsable del daño neurológico en ELA. ${ }^{26}$

Cabe señalar que el fenotipo puede diferir según la región del gen que las mutaciones afecten. No existe una correlación genotipo-fenotipo muy clara, por las diferencias de presentación entre los casos familiares y esporádicos, e intrafamiliares con la misma mutación, ${ }^{22-26}$ lo que se atribuye a la expresividad variable 0 a genes modificadores. ${ }^{10}$

Se ha postulado a ELA como una enfermedad conformacional, porque las mutaciones en SOD1 llevan a cambios en la estructura de la enzima y afectan su actividad catalítica (actividad menor de $50 \%$ ), así como a haploinsuficiencia. ${ }^{22-23}$ Por otra parte, la acumulación de esta proteína con defectos en el 
plegamiento está relacionada con mutaciones, se traduce en ganancia de la función con un efecto tóxico, denominado efecto dominante negativo. ${ }^{26-27}$

Son limitados los estudios de mutagénesis dirigida que analizan la haploinsuficiencia y el efecto dominante negativo; en futuros trabajos será importante investigarlos para identificar el efecto clínico del cambio de aminoácido en la estructura del sitio activo, la región alostérica y otros dominios funcionales de SOD1.

\section{Avances en la genética de ELA}

Mediante secuenciación de nueva generación, en población francesa se encontró $20 \%$ de casos familiares, con una frecuencia de $50 \%$ para las mutaciones de SOD $1 .{ }^{28}$ Mientras que en población inglesa se encontraron las siguientes mutaciones: p.C256G, p.G229T, p.A272C, p.A305G, p.C310T, p.G335A, p.T341C y p.A403G. ${ }^{29}$ Recientemente en una mujer con ELA de ancestría húngara se encontró una nueva mutación c.791A $>\mathrm{G}$ en el gen SETX. ${ }^{30}$ En población con esa misma ancestría se han reportado nuevas mutaciones en el gen SOD1 en casos esporádicos; p.K91R/X, p.V14M, p.D90A, p.L144F y p.L91R/X8. ${ }^{31}$ También se ha encontrado la expansión anormal de (GGGGCC)n del gen C9ORF72 hasta en $30 \%$ de casos ELAE. ${ }^{31-32}$ Los genes SOD1 y C9orf72 tienen la contribución más importante en la patogénesis de la enfermedad. Actualmente, los nuevos blancos en la terapia de ELA se centran en los disturbios de la homeostasis de las proteínas, alteraciones de la biología de las proteínas que se unen al ARN, así como los defectos en la dinámica del citoesqueleto. ${ }^{33}$

\section{Fronteras de la investigación en la ELA}

Los nuevos retos en el estudio de ELA son el efecto de las modificaciones epigenéticas, que podrían arrojar luz sobre la edad de inicio, presentación familiar o severidad, para facilitar la identificación de terapias eficaces, diagnóstico precoz y potenciales intervenciones terapéuticas en etapas tempranas. ${ }^{34}$ En Latinoamérica no hay investigaciones con información sobre la genética de ELA. A nivel inmunológico se ha explorado el papel de 19 citocinas, entre ellas la adipsina, adiponectina, IL-4 e IL-6 en relación con la gravedad clínica y duración de la enfermedad. La adipsina fue encontrada elevada en líquido cefalorraquídeo; la adiponectina mostró una tendencia hacia concentraciones más altas. ${ }^{35-36}$ Ciertamente son necesarios más estudios epidemiológicos sobre estas interleucinas para establecer su valor pronóstico. Esta evidencia puede sugerir que las variantes en los genes que codifican para estas proteínas pueden modificar la expresión clínica de ELA. Entre los estudios clínicos destacan un reporte de cuatro casos de ELA familiar juvenil y supervivencia prolongada. ${ }^{37-39}$ Recientemente, nuestro grupo de trabajo reportó un caso de ELA en México, positivo para una mutación en el gen MT-CYTB. ${ }^{40}$

\section{Conclusiones}

ELA es una enfermedad genética compleja, lo que se refleja en sus diferentes patrones de herencia, componente mutacional, alta penetrancia, edad de inicio variable, múltiples vías metabólicas alteradas simultáneamente en los pacientes con esta enfermedad. Por otra parte, la mayoría de mutaciones encontradas en SOD1 son responsables principalmente de ELAF y de un tercio de los casos de ELAE. Existen otros genes que deben ser explorados como causa, sobre todo en ELAF: C9orf72 y SETX. A nivel clínico en México, las citocinas proinflamatorias podrían ser marcadores pronósticos, aunque será necesario validarlos.

\section{Conflicto de interés}

No existieron conflictos de interés por parte de los autores.

\section{Agradecimientos}

A Conacyt, por la beca de estancia sabática nacional al doctor Sergio A. Ramírez García, de la Universidad de la Sierra Sur.

\section{Bibliografía}

1. Woolley SC, Rush BK. Considerations for clinical neuropsychological evaluation in amyotrophic lateral sclerosis. Arch Clin Neuropsychol. 2017;32:906-916

2. Couratier P, Marin B, Lautrette G, Nicol M, Preux PM. Epidemiology, clinical spectrum of ALS and differential diagnoses. Presse Med. 2014:43:538-548.

3. Volk AE, Weishaupt JH, Andersen PM, Ludolph AC, Kubisch C. Current knowledge and recent insights into the genetic basis of amyotrophic lateral sclerosis. Med Genet. 2018;30:252-258.

4. Rohrer JD, Isaacs AM, Mizielinska S, Mead S, Lashley T, Wray S, et al. C9orf72 expansions in frontotemporal dementia and amyotrophic lateral sclerosis. Lancet Neurol. 2015;14:291-301.

5. Bunton-Stasyshyn RK, Saccon RA, Fratta P, Fisher EM. SOD1 function and its implications for amyotrophic lateral sclerosis pathology: new and renascent themes. Neuroscientist. 2015;21:519-529.

6. Jones CT, Brock DJ, Chancellor AM, Warlow CP, Swingler RJ. Cu/Zn superoxide dismutase (SOD1) mutations and sporadic amyotrophic lateral sclerosis. Lancet. 1993;342:1050-1051. 
7. Schmitt ND, Agar JN. Parsing disease-relevant protein modifications from epiphenomena: perspective on the structural basis of SOD1-mediated ALS. J Mass Spectrom. 2017;52:480-491.

8. Kikugawa K, Nakano R, Inuzuka T, Kokubo Y, Narita Y, Kuzuhara S, et al. A missense mutation in the SOD1 gene in patients with amyotrophic lateral sclerosis from the Kii Peninsula and its vicinity, Japan. Neurogenetics. 1997;1:113-115.

9. Zhang CC, Zhu JX, Wan Y, Tan L, Wang HF, Yu JT, Tan L. Meta-analysis of the association between variants in MAPT and neurodegenerative diseases. Oncotarget. 2017;8:44994-45007.

10. Morello G, Spampinato AG, Cavallaro S. molecular taxonomy of sporadic amyotrophic lateral sclerosis using disease-associated genes. Front Neurol. 2017;8:152

11. Battistini S, Giannini F, Greco G, Bibbò G, Ferrera L, Marini V, et al. SOD1 mutations in amyotrophic lateral sclerosis. Results from a multicenter Italian study. J Neurol. 2005;252:782-788.

12. Chiò A, Mazzini L, D'Alfonso S, Corrado L, Canosa A, Moglia C, et al. The multistep hypothesis of ALS revisited: the role of genetic mutations. Neurology. 2018;91:e635-e642.

13. Peters OM, Ghasemi M, Brown RH. Emerging mechanisms of molecular pathology in ALS. J Clin Invest. 2015;125:1767-1779.

14. Salem K, McCormick ML, Wendlandt E, Zhan F, Goel A. Copperzinc superoxide dismutase-mediated redox regulation of bortezomib resistance in multiple myeloma. Redox Biol. 2015;4:23-33.

15. Venkataramani V, Doeppner TR, Willkommen D, Cahill CM, Xin Y, Ye G, et al. Manganese causes neurotoxic iron accumulation via translational repression of Amyloid precursor protein and $\mathrm{H}$-Ferritin. J Neurochem. 2018;147:831-848.

16. Majzúnová $M, P a k a n o v a ́ ~ Z$, Kvasnička $P$, Bališ $P$, Čačányiová $S$, Dovinová I. Age-dependent redox status in the brain stem of NO-deficient hypertensive rats. J Biomed Sci. 2017;24:72.

17. Popović-Bijelić A, Mojović M, Stamenković S, Jovanović M, Selaković V, Andjus $\mathrm{P}$, et al. Iron-sulfur cluster damage by the superoxide radical in neural tissues of the SOD1(G93A) ALS rat model. Free Radic Biol Med. 2016;96:313-322.

18. D'Ambrosi N, Cozzolino M, Carrì MT. Neuroinflammation in Amyotrophic lateral sclerosis: role of redox (dys) regulation. Antioxid Redox Signal. 2018;29:15-36.

19. Pardo CA, Xu Z, Borchelt DR, Price DL, Sisodia SS, Cleveland DW. Superoxide dismutase is an abundant component in cell bodies, dendrites, and axons of motor neurons and in a subset of other neurons. Proc Natl Acad Sci U S A. 1995;92:954-958.

20. Carocci A, Catalano A, Sinicropi MS, Genchi G. Oxidative stress and neurodegeneration: the involvement of iron. Biometals. 2018;31:715-735.

21. Andersen PM, Sims KB, Xin WW, Kiely R, O'Neill G, Ravits J, et al. Sixteen novel mutations in the $\mathrm{Cu} / \mathrm{Zn}$ superoxide dismutase gene in amyotrophic lateral sclerosis: a decade of discoveries, defects and disputes. Amyotroph Lateral Scler Other Motor Neuron Disord. 2003:4:62-73.

22. Deng HX, Hentati A, Tainer JA, Iqbal Z, Cayabyab A, Hung WY, et al. Amyotrophic lateral sclerosis and structural defects in $\mathrm{Cu}, \mathrm{Zn}$ superoxide dismutase. Science. 1993;261:1047-1051.

23. Abe K, Aoki M, Ikeda M, Watanabe M, Hirai S, Itoyama $Y$, et al. Clinical characteristics of familial amyotrophic lateral sclerosis with $\mathrm{Cu} / \mathrm{Zn}$ superoxide dismutase gene mutations. J Neurol Sci. 1996;136:108-116.
24. Eisen A, Mezei MM, Stewart HG, Fabros M, Gibson G, Andersen PM, et al. SOD1 gene mutations in ALS patients from British Columbia, Canada: clinical features, neurophysiology and ethical issues in management. Amyotroph Lateral Scler. 2008;9:108-119.

25. Alavi A, Nafissi S, Rohani M, Zamani B, Sedighi B, Shamshiri H, et al., Genetic analysis and SOD1 mutation screening in Iranian amyotrophic lateral sclerosis patients. Neurobiol Aging. 2013;34:1516.e1-8.

26. Battistini S, Ricci C, Giannini F, Calzavara S, Greco G, Del Corona A et al. G41S SOD1 mutation: a common ancestor for six ALS Italian families with an aggressive phenotype. Amyotroph Lateral Scler. 2010:11:210-215.

27. Alemasov NA, Ivanisenko NV, Taneja B, Taneja V, Ramachandran S, Ivanisenko VA. Improved regression model to predict an impact of SOD1 mutations on ALS patients survival time based on analysis of hydrogen bond stability. J Mol Graph Model. 2018;86:247-255.

28. Corcia P, Couratier P, Blasco H, Andres CR, Beltran S, Meininger V, et al. Genetics of amyotrophic lateral sclerosis. Rev Neurol (Paris). 2017;173:254-262.

29. Morgan S, Shatunov A, Sproviero W, Jones AR, Shoai M, Hughes D, et al. A comprehensive analysis of rare genetic variation in amyotrophic lateral sclerosis in the UK. Brain. 2017;140:1611-1618.

30. Tripolszki K, Török D, Goudenège $D$, Farkas $K$, Sulák A, Török N, et al. High-throughput sequencing revealed a novel SETX mutation in a Hungarian patient withamyotrophic lateral sclerosis. Brain Behav. 2017;7:e00669.

31. Tripolszki K, Csányi B, Nagy D, Ratti A, Tiloca C, Silani V, et al. Genetic analysis of the SOD1 and C9ORF72 genes in Hungarian patients with amyotrophic lateral sclerosis. Neurobiol Aging. 2017;195:e1-195.e5

32. Martin S, Al-Khleifat A, Al-Chalabi A. What causes amyotrophic lateral sclerosis? F1000Res. 2017;8:371.

33. Ghasemi M, Brown RH. Genetics of amyotrophic lateral sclerosis. Cold Spring Harb Perspect Med. 2018;8:pii.

34. Jiménez-Pacheco A, Franco JM, López S, Gómez-Zumaquero JM, Magdalena-Leal-Lasarte $M$, Caballero-Hernández DE, et al. Epigenetic mechanisms of gene regulation in amyotrophic lateral sclerosis. Adv Exp Med Biol. 2017;978:255-275.

35. Martínez HR, Escamilla CE, Camara CR, González MT, Tenorio JM, Hernández M. CSF concentrations of adipsin and adiponectin in patients withamyotrophic lateral sclerosis. Acta Neurol Belg. 2017;117(4):879-883.

36. Martínez HR, Escamilla CE, TenorioJM, Gómez D, Jaime JC, OIguín-LA, et al. Altered CSF cytokine network in amyotrophic lateral sclerosis patients: a pathway-based statistical analysis. Cytokine. 2017:90:1-5.

37. Martínez HR, Parada JD, Meza ME, González MT, Moreno CJ. Esclerosis lateral amiotrófica. Contribución de la neurología mexicana de 1998 a 2014. Rev Mex Neuroci. 2014:15:355-362.

38. Arriada N, Ríos $\mathrm{C}$, Otero-Siliceo $\mathrm{E}$, Corona-Vázquez T. ALS in a secluded region in Mexico possibly related to lead toxicity. Arch Neurociences. 2000;5:2-5.

39. Bresch S, Delmont E, Soriani MH, Desnuelle C. Electrodiagnostic criteria for early diagnosis of bulbar-onset ALS: a comparison of El Escorial, revised El Escorial and Awaji algorithm. Rev Neurol (Paris). 2014;170:134-139.

40. Cervantes I, Ramírez S, García D. Amyotrophic lateral sclerosis positive for mutation in the CYTB gene and negative for SOD1 and ATXN2. Rev Fac Med. 2017;65:377-378. 